\title{
Acute effects of riluzole and retigabine on axonal excitability in patients with ALS: a randomized, double-blind, placebo-controlled, cross-over trial
}

Maria O Kovalchuk ${ }^{1 *}$, M.D., Jules AAC Heuberger ${ }^{2^{*}}$, MSc, Boudewijn THM Sleutjes ${ }^{1}$, Ph.D., Dimitrios

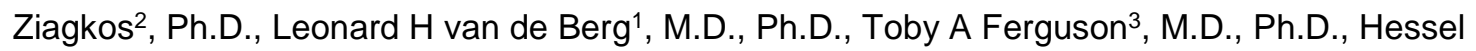
Franssen1\#, M.D., Ph.D., Geert Jan Groeneveld²\#, M.D., Ph.D.

1 University Medical Center Utrecht, Department of Neurology, Utrecht, the Netherlands ${ }^{2}$ Centre for Human Drug Research, Leiden, the Netherlands ${ }^{3}$ Biogen, Department of Neurology Research and Early Clinical Development, Cambridge, United States

*Both authors contributed equally to this work

\#Both authors contributed equally to this work

Corresponding author: Jules Heuberger, MSc

Address: Zernikedreef 8, $2333 \mathrm{CL}$, Leiden, The Netherlands

E-mail: jheuberger@chdr.nl

Tel: +31715246471

Fax: +31715246499

Number of figures: 4

Number of tables: 3

Number of references: 44

Keywords: Amyotrophic lateral sclerosis, Nerve excitability, Neurodegeneration biomarkers 


\section{Funding}

Biogen funded the study. Toby Ferguson is an employee of Biogen.

\section{Potential Conflicts of Interest}

Ms. Kovalchuk reports grants from European Federation of Neurological Societies, grants from

Prinses Beatrix SpierFonds, outside the submitted work.

Mr. Heuberger reports grants from Biogen, during the conduct of the study.

Dr. Sleutjes reports grants from Prinses Beatrix Spierfonds, outside the submitted work.

Dr. Ziagkos reports grants from Biogen, during the conduct of the study.

Dr. van den Berg reports personal fees from Biogen, personal fees from Cytokonetics, personal fees from Orion, grants from Centre for Human Drug Research, during the conduct of the study.

Dr. Ferguson is employee for Biogen, which funded the study.

Dr. Franssen reports grants from Biogen, during the conduct of the study; grants from Prinses Beatrix Spierfonds, personal fees from Shire, outside the submitted work.

Dr. Groeneveld reports grants from Biogen, during the conduct of the study. 


\section{Abstract}

Increased excitability of motor neurons in patients with amyotrophic lateral sclerosis may be a relevant factor leading to motor neuron damage. This randomized, doubleblind, 3-way cross-over, placebo-controlled study evaluated peripheral motor nerve excitability-testing as biomarker of hyperexcitability and assessed effects of riluzole and retigabine in 18 patients with amyotrophic lateral sclerosis. We performed excitability-testing at baseline, and twice after participants had received a single dose of either $100 \mathrm{mg}$ riluzole, $300 \mathrm{mg}$ retigabine or placebo. Between- and within-day repeatability was at least acceptable for 14 out of 18 recorded excitability variables. No effects of riluzole on excitability-testing were observed, but retigabine significantly decreased strength-duration time-constant (9.2\%) and refractoriness at $2 \mathrm{~ms}(10.2$ percentage points) compared to placebo. Excitability-testing was shown to be a reliable biomarker in patients with amyotrophic lateral sclerosis, and the acute reversal of previously abnormal variables by retigabine justifies long-term studies evaluating impact on disease progression and survival. 


\section{Introduction}

Amyotrophic lateral sclerosis (ALS) is a progressive and fatal neurodegenerative disease characterized by ongoing loss of motor neurons. In ALS the unique phenomenon of increased membrane excitability in both peripheral and central motor neurons can be observed, presenting clinically as fasciculations, muscle cramps, hyper-reflexia, and spasticity.(1) Excitability-testing is a neurophysiological tool which allows non-invasive assessment of axolemmal voltage-gated ion-channel activity in motor axons of a peripheral nerve. In ALS, it showed evidence of increased persistent sodium-conductance and reduced potassium-conductance, both of which may contribute to axonal hyperexcitability and fasciculation.(2-6) Furthermore, presence of increased persistent sodium-conductance was shown to be correlated with more rapid functional decline and shorter survival, $(7,8)$ and presence of fasciculation with shorter survival.(9) Because increased membrane excitability in ALS may be a relevant step in the cascade leading to structural damage of motor neurons,(10) early identification of hyperexcitable motor neurons may provide an argument for initiating neuroprotective intervention. $(7,11)$ Retigabine, a potassiumchannel activator, was shown to reduce increased cellular membrane excitability and improve cell survival in an in vitro model of ALS.(12) Riluzole, the only registered drug for treatment of ALS, partially normalized some excitability variables in peripheral and cortical motor neurons of patients with ALS.(13) Modulation of hyperexcitability could therefore serve as a proof-of-pharmacology biomarker to assess effects of therapeutic interventions in ALS. The present study aimed to validate motor nerve excitability-testing of the median nerve as a biomarker of hyperexcitability and assessed pharmacodynamic effects of retigabine and riluzole in patients with ALS. 


\section{Results}

The interim analysis showed at least acceptable repeatability (alpha $>0.7$ ) for all five pre-determined variables (data not shown). Therefore, in total, eighteen patients with ALS were included and all subjects completed the study (Figure 1), with recruitment running from October 2015 to December 2016, and the last follow-up phone call taking place in April 2017. Baseline characteristics are displayed in Table 1. Participants tolerated the study and treatments well. One subject did not complete the $6 \mathrm{~h}$ measurement of the first visit (riluzole occasion) because of adverse events consisting of nausea and vomiting due to a migraine attack. It was considered unlikely that this was related to the study treatment. There were 15 adverse events in the retigabine-arm, 14 in the riluzole-arm, and 6 in the placebo-arm. All events were grade 1-2 and none was reported more than twice per arm, except for dizziness (reported 3 times in the retigabine-arm) and somnolence (reported 7 times in the retigabine and 3 times in the placebo-arm).

\section{Pharmacokinetics}

Pharmacokinetic analysis showed mean $\mathrm{C}_{\max }$ for riluzole of $343 \mathrm{ng} / \mathrm{mL}$ (range: $102-$ $646 \mathrm{ng} / \mathrm{mL})$ and for retigabine of $604 \mathrm{ng} / \mathrm{mL}(271-997 \mathrm{ng} / \mathrm{mL})$, both on average at 1 hour post-dose (riluzole range: $0.5-3$ hours; retigabine range: $0.5-4$ hours), see Figure 2. Variability in $\mathrm{C}_{\max }$ was moderate with a coefficient of variation (CoV) of $48 \%$ for riluzole and $36 \%$ for retigabine. $T_{\max }$ was more variable for retigabine (CoV $90 \%$ ) than for riluzole (CoV 66\%). Mean riluzole concentration before dosing for all treatment periods combined was $12 \mathrm{ng} / \mathrm{mL}$ (range: $<1-75.7 \mathrm{ng} / \mathrm{mL}$ ), and $10 \mathrm{ng} / \mathrm{mL}$ $(1.9-22.3 \mathrm{ng} / \mathrm{mL})$ for the placebo treatment period. 


\section{Baseline electrophysiological characteristics and test-retest reliability}

Baseline electrophysiological characteristics, as well as repeatability for excitabilitytesting are presented in Table 2. Repeatability between each baseline measurement (inter-occasion), as determined by Cronbach's alpha, showed that the majority of variables had an acceptable (Cronbach's alpha $>0.7$ ) to excellent repeatability. Only accommodation half-time showed Cronbach's alpha much lower than 0.7 ; rheobase, SDTC and threshold for a target CMAP of $50 \%$ were just below the threshold of Cronbach's alpha $>0.7$. Within the placebo visit (intra-occasion), repeatability was just below acceptable for SDTC, and well below 0.7 for accommodation half-time, but good to excellent for all other parameters. When compared to controls, the excitability variables obtained in ALS patients at the pre-dose visit showed greater changes in TEd40-60 (53.0 \pm (SEM) $1.2 \%$ versus $47.3 \pm 0.9 \%, p<0.001)$ and TEd90-100 (48.8 $\pm 1.6 \%$ versus $44.1 \pm 0.7 \%, p=0.008)$, longer accommodation halftime (38.7 $\pm 1.0 \mathrm{~ms}$, versus $35.8 \pm 0.7 \mathrm{~ms}, \mathrm{p}=0.02)$, increased superexcitability ($27.4 \pm 2.1 \%$, versus $20.5 \pm 1.0 \%, p=0.004)$, and decreased subexcitability $(11.9 \pm$ $1.0 \%$ versus $16.7 \pm 1.3 \%, p=0.006)$. Other variables, including SDTC $(0.46 \pm 0.02$ ms versus $0.45 \pm 0.01 \mathrm{~ms}, p=0.86$ ), were not significantly different.

\section{Effects of riluzole and retigabine on motor nerve excitability}

Figure $3 \mathrm{i}$ shows the mean excitability recordings at pre-dose and after a single dose of $100 \mathrm{mg}$ riluzole at 1.5 and 6 hours. No statistically significant effects were observed for riluzole on any of the excitability measures compared to placebo. Figure 3ii shows the mean excitability recordings at pre-dose and after a single dose of 300 $\mathrm{mg}$ retigabine at 1.5 and 6 hours. Significant treatment effects were observed for retigabine, showing the following effects compared to placebo: increase in 
hyperpolarizing I/V-slope (21.7\%), resting I/V-slope (6.1\%), minimum I/V-slope (8.5\%), rheobase (28.0\%), threshold for a target CMAP of $50 \%(25.0 \%)$, accommodation half-time $(3.15 \mathrm{~ms})$, decrease in SDTC $(9.2 \%)$, refractoriness at $2 \mathrm{~ms}$ (10.2 percent point, or arithmetic difference) and refractory period $(0.17 \mathrm{~ms})$ (Table 3 , Figure 4).

\section{Predictive value of excitability measures}

None of the excitability-variables at baseline showed a significant correlation with clinical deterioration by a functional decline in ALSFRS-R score between baseline and 3 months. 


\section{Discussion}

Excitability-testing was shown to produce repeatable results in patients with ALS, both within and between visits, for all 18 variables except accommodation half-time. Although riluzole did not show effects, retigabine had significant effects on several excitability-variables when compared to placebo. No correlation between ALSFRS-R and excitability-variables was found.

\section{Electrophysiological characteristics and variability}

Inter-subject variability for parameters such as CMAP, refractoriness at $2 \mathrm{~ms}$, rheobase, I/V-slopes, super- and subexcitability was relatively high (CV of 27-63\%), which may likely be related to differences in the disease state of the patients. Intrasubject variability is, therefore, more informative on variability, and CVs were indeed much smaller. The statistical model-based estimate of the intra-subject CV for all parameters ranged from $4-23 \%$, and was for most CVs very similar to statistical model based estimate found in healthy volunteers.(14) Only the CVs of accommodation half-life, TEd90-100, TEh90-100 and hyperpolarizing I/V slope were more than 1.5 times greater than found by Tomlinson et al.(14)

\section{Pharmacokinetics}

The $\mathrm{C}_{\max }$ of riluzole for all but three subjects (mean $343 \mathrm{ng} / \mathrm{mL}$, range 102-646 $\mathrm{ng} / \mathrm{mL}$ ) and of retigabine for all subjects (mean $604 \mathrm{ng} / \mathrm{mL}$, range 271-997 $\mathrm{ng} / \mathrm{mL}$ ) was above the approximate therapeutic levels of $173 \mathrm{ng} / \mathrm{mL}$ for riluzole(15) and 250 $\mathrm{ng} / \mathrm{mL}$ for retigabine(16) as expected with the selected supra-therapeutic doses. Mean plasma concentration of riluzole before dosing for all treatment periods was 12 $\mathrm{ng} / \mathrm{mL}$ (range $<1-75.7 \mathrm{ng} / \mathrm{mL}$ ) as could be expected after approximately $24 \mathrm{~h}$ - 
washout of riluzole; thereby levels were approximately 30 times lower than at $\mathrm{C}_{\max }$ during the riluzole period. As the riluzole concentration exerting $50 \%$ of the maximal effect $\left(\mathrm{IC}_{50}\right)$ on voltage gated sodium channels is approximately $0.3 \mu \mathrm{M}$ (or 70 $\mathrm{ng} / \mathrm{mL}),(17)$ the mean post-washout level of $12 \mathrm{ng} / \mathrm{mL}$ is unlikely to have affected sodium channel function. Two subjects had significantly higher riluzole baseline levels at one visit (with 63.2 and $75.7 \mathrm{ng} / \mathrm{mL}$ ), possibly due to not having followed the instruction to omit their regular evening dose of riluzole. Both instances occurred during retigabine treatment periods. It is, however, unlikely that this influenced the observed effects in the retigabine treatment arm, especially as no effect of riluzole was observed in the riluzole treatment arm. Sensitivity analysis, with exclusion of these occasions did not produce a different outcome, with one exception: that retigabine effects on minimum I/V-slope and resting I/V-slope did not reach significance (borderline) (data not shown).

\section{Effects of retigabine on peripheral nerve excitability}

In human peripheral motor nerve, five types of voltage-gated potassium channels have been described depending on their gating modes, activation-deactivation time and conductance, with a large overlap between their kinetic properties. $(18,19)$ These five types give rise to three types of potassium-currents on single axon recordings: fast, intermediate and slow. Slowly activating potassium channels of the axonal membrane belong to Kv7.2-Kv7.5 subtypes, coded by the KCNQ genes. $(20,21)$ In vitro studies and animal models of epilepsy and pain showed that retigabine hyperpolarizes resting axonal membrane potential by inducing these potassium channel subtypes to open which, in turn, enhances outward slow potassium currents and produces a hyperpolarizing shift of the half-activation potential of these 
channels. $(22,23)$ Excitability-variables assessed after potassium channel activators such as retigabine and flupirtine administration are, therefore, expected to reflect either potassium channel activation or the resulting hyperpolarization of resting membrane potential. $(20,21)$

In our study, a single dose of retigabine resulted in significant changes in various axonal excitability-parameters. Strength duration properties showed a decrease in SDTC and increase in rheobase. Both can be explained by either hyperpolarization of resting membrane potential (since hyperpolarization decreases nodal persistent sodium current), $(24,25)$ or a decrease in nodal electrical capacitance. As it is unlikely that retigabine induces histological changes resulting in decreased nodal capacitance, hyperpolarization of resting membrane potential is the most likely mechanism. Membrane hyperpolarization also results in increased threshold current needed to evoke a target CMAP of $50 \%$ of its maximum amplitude, similar to the findings in previous studies.(26) In the current-threshold relationship, a steeper hyperpolarizing I/V slope was found, producing a larger inward rectification, which is also expected to be enhanced when the axonal membrane potential becomes more hyperpolarized. Furthermore, in the present study, retigabine reduced refractoriness at $2 \mathrm{~ms}$ and refractory period, which is consistent with a decrease in sodium-channel inactivation due to membrane hyperpolarization. $(27,28)$ Shortening of the refractory period may also be due to early repolarization of the action potential. Early repolarization by retigabine has been observed previously as a result of membrane hyperpolarization induced by a hyperpolarizing shift of the voltage dependence of slow potassium channels.(29) This effect is most likely due to the greater number of potassium channels open at hyperpolarized membrane potentials. As riluzole has 
been previously shown to reduce refractoriness at $2 \mathrm{~ms}$,(13) this observed effect of retigabine might be beneficial in ALS.

In the recovery cycle, retigabine did not induce significant changes in superexcitability and late subexcitability; this could be due to a plasma concentration being too low to induce detectable effects, as shown in a study with flupirtine.(30) In our study, we determined subexcitability after only a single supramaximal preconditioning pulse, where previous studies applied multiple pre-conditioning supramaximal pulses, $(26,31)$ known to enhance late after-hyperpolarization, increasing subexcitability (32) and the sensitivity to detect treatment-induced changes.

Retigabine did not normalize any of the parameters that were found to be significantly different from healthy controls in our study. It did however, change SDTC in the direction of normalization, a variable that has previously been shown to be abnormally increased in patients with ALS. $(2,4-6)$ There was no significant correlation between retigabine concentration and effects on excitability variables (not shown), however, the appropriate approach would be to develop a population pharmacokinetic-pharmacodynamic model, which will be explored in the future. In any case, the chosen study design and statistical analysis ensure that the observed effects are induced by retigabine and cannot for example be explained by disease progression.

\section{Effects of riluzole on peripheral nerve excitability}

In our study, a single dose of riluzole had no significant effects on excitabilityvariables. It is possible that the period of riluzole administration in our study was too short, since riluzole administration of, on average, 7 weeks in patients with ALS 
decreased refractoriness at $2 \mathrm{~ms}$ and superexcitability.(13) Nevertheless, we expected an effect on excitability-variables after a single-dose of riluzole, as it inhibits persistent sodium currents and shifts the voltage dependence of sodium channel inactivation in a negative direction.(33) Another possible explanation of the lack of effect in our study may be related to the low riluzole concentrations that were still present during the baseline measurement, despite the preceding washout of standard riluzole treatment. This seems, however, unlikely because concentrations were approximately 5 times below the $\mathrm{IC}_{50}$ for voltage-gated sodium channels and because no effect of riluzole on excitability-variables was found in the riluzole-naïve patient of our study. Also, riluzole might have been less effective due to persistent sodium channels remaining in an open state.(13) Finally, there are indications that riluzole might lose its efficacy in later stages of the disease, $(34,35)$ and our patients had been diagnosed, on average, more than a year prior to starting the study. A hypothesis for this loss of efficacy is that upregulation of efflux transporters in disease-affected regions,(36) such as P-glycoprotein (PGP) and breast cancer resistance protein (BCRP), would lead to very low concentrations at the target site.

\section{Relation with disease progression}

A relation between SDTC and ALSFRS-R(8) decline and SDTC and survival(7) have been reported, but in the current study no correlation was observed between any of the excitability parameters and disease progression as measured by a change in ALSFRS-R. This could be due to the shorter follow-up (3 months compared to 6 months) and/or lower number of subjects in our study (18 versus 60$)$. When the follow-up was extended in our study in an ad-hoc analysis to, on average, 14.5 months (range 5.8 to 20.2 months) after baseline ALSFRS-R, again no significant 
correlation was found between excitability parameters and change in ALSFRS-R normalized for interval (time between baseline and second questionnaire) (data not shown).

\section{Limitations}

The most important limitation of our study was a lack of complete washout of standard riluzole treatment as described in the previous sections. As all but one participant were men, one should be cautious in extrapolating the results to female patients. Four (22\%) of the enrolled patients had a familial history of ALS and therefore probably a hereditary form in which the pathophysiological mechanism might differ from that in patients with sporadic ALS.

\section{Clinical implications}

This study shows that a single dose of retigabine has a greater effect on peripheral nerve excitability than a single dose of riluzole, the current registered treatment for ALS. Previous studies showed that a prolonged SDTC is related to more rapid disease progression and shorter survival. $(7,8)$ Although SDTC in our ALS patients was not statistically different from that in healthy controls, retigabine induced shortening of this variable. Long-term retigabine administration may, therefore, reverse the increased persistent sodium current underlying SDTC prolongation which was suggested to induce hyper-excitability and motor neuron death.(8, 12) If, in the future, peripheral nerve excitability proves to be predictive of clinical outcome, it might be a very useful, non-invasive biomarker to test for potential treatments for ALS, and measure treatment efficacy on a much shorter basis compared to the sensitivity of the ALSFRS-R or survival measures. 


\section{Methods}

\section{Subjects}

Eighteen patients with ALS, aged between 18 and 80 years were enrolled in the study, which was performed at the University Medical Center Utrecht in Utrecht, The Netherlands in collaboration with the Centre for Human Drug Research, Leiden, The Netherlands. Patients were recruited via the department's patient database, through advertisements, and the newsletters of the "Vereniging Spierziekten Nederland" (VSN), the organization for patients with neuromuscular disorders in the Netherlands. All patients gave written informed consent prior to any study-related activity, after which a screening visit evaluated eligibility. Main inclusion criteria were: mastery of the Dutch language, diagnosis of definite, probable, or probable laboratory-supported ALS according to the revised El Escorial criteria of 1998,(37) fasciculations in the lower arm to be used for excitability measurements observed by the treating neurologist, CMAP of the abductor pollicis brevis muscle in the arm with fasciculations exceeding $1 \mathrm{mV}$, as well as no history of diabetes, neuropathy, or neuromuscular disorders other than ALS, carpal tunnel syndrome, trauma to the upper extremities or other orthopedic conditions that might affect the electrophysiological measurements, and no medication that might affect electrophysiological measurements, other than that used in the study. During the trial, the inclusion criterion for fasciculations was modified to include subjects with fasciculations anywhere in the arm, not only in the lower arm, and an exclusion for history of alcohol or drug dependence was removed. These changes were made in order to facilitate patient recruitment and were not considered to impact the study validity. 


\section{Experimental design}

We performed a randomized, double blind, 3-way cross-over, placebo controlled study of the test-retest reliability of peripheral motor nerve excitability and the effects of oral retigabine and riluzole on these measurements in patients with ALS. Visits were scheduled a week apart in order to allow a sufficiently long washout of riluzole and retigabine. Eligible subjects arrived at the research unit on the morning of a treatment visit, and after passing a brief re-eligibility and health check, they underwent baseline excitability-testing. Capsules with medication were then swallowed with water on an empty stomach, after which regular blood samples were collected for pharmacokinetic analysis. Subjects were required to remain fasted until 2.5 hours after dosing, although water was allowed. At 1.5 and 6 hours after dosing excitability-testing was repeated. Blood samples for pharmacokinetic analysis were taken pre-dose and 0.5, 1, 1.5, 2, 2.5, 3, 4, 6 and 7 hours after dosing. After the last blood sample the subject was discharged.

Since riluzole is the standard treatment for ALS patients (50 mg b.i.d.), we instituted a one-day washout of riluzole before each dosing occasion to minimize the effect of the drug on excitability variables. Although the half-life of riluzole is approximately 915 hours(38) and not all of the drug was expected to have washed out of the system after 24 hours, it was deemed unethical to have a longer washout period as this might have impacted the efficacy of the treatment, and the willingness of the patients to participate in the study.

The study was approved by the Independent Ethics Committee of the Foundation 'Evaluation of Ethics in Biomedical Research' (Stichting Beoordeling Ethiek Biomedisch Onderzoek), Assen, The Netherlands. The study was registered in the 
Dutch Trial Registry (Nederlands Trial Register, NTR) under study number NTR6278 and was carried out between November 2015 and April 2017.

\section{Treatment, randomization and masking}

Subjects received a single dose of $100 \mathrm{mg}(2 \times 50 \mathrm{mg})$ riluzole (Rilutek, Sanofi, Paris, France), 300 mg (100 and $200 \mathrm{mg}$ ) retigabine (Trobalt, Glaxo Smith Kline, Brentford, UK) as encapsulated tablets or matching placebo capsules. Subjects were randomly assigned to a treatment order, with a balanced design using a code generated by an un-blinded statistician who was otherwise not involved in the execution of the study. A masked physician enrolled patients into the study. Until study closure the treatment codes were only available to this statistician and the Leiden University Medical Center (LUMC) pharmacy, which distributed the study agents.

\section{Motor nerve excitability-testing}

Motor excitability was measured in the median nerve at the wrist. The setup consisted of Viking IV EMG apparatus (Nicolet Biomedical, Inc., Madison, Wisconsin), coupled to a computer (PCI-6221, National instruments) running QTRAC-S software (TRONDNF, version 19-06-2015, Institute of Neurology, Queen Square, London, UK) and an isolated bipolar constant current stimulator (DS5, Digitimer, UK model D185-HB4). The median nerve was stimulated at the wrist via non-polarizable surface electrodes (cathode at the wrist; anode $10 \mathrm{~cm}$ proximal over the radial side of the forearm). The thenar CMAP was recorded by surface electrodes in a belly-tendon montage. The distance between active recording electrode and stimulating cathode was $7 \mathrm{~cm}$. The median nerve was warmed to $37^{\circ} \mathrm{C}$ by wrapping the forearm and hand for 30 minutes in a warm water blanket through which water at 
$37^{\circ} \mathrm{C}$ flowed constantly (Cincinatti Sub-zero Norm-O-Temp with Cincinatti Sub-zero maxi-therm lite infant hyper-hypothermia blanket for single patient use).(39) During excitability-testing the forearm and hand were kept in the blanket with flowing water at $37^{\circ} \mathrm{C}$ in order to maintain a constant nerve temperature. Skin temperature was continuously monitored by means of a sensor near the stimulating cathode. Distal motor latency (DML) was measured every 1.6 seconds during each excitabilitytesting in order to check if changes in nerve temperature resulted in conduction changes. DML was defined as the point where the CMAP deviated by $10 \%$ of its amplitude from baseline to peak. These procedures were based on previous studies.(39-41)

To examine axonal excitability parameters a specific sequence of conditioning and test stimuli were applied to the nerve. Conditioning stimuli were constant currents that either slightly depolarized resting membrane potential, slightly hyperpolarized resting membrane potential, or induced nerve action potentials. Threshold was defined as the test-stimulus current needed for a target CMAP of $40 \%$ of its maximum amplitude.

Each excitability-test consisted of: stimulus response (SR) curve (relation between stimulus current and response amplitude), charge-duration (Qt) relation (relation between stimulus charge and stimulus duration), threshold electrotonus (time course of threshold changes during a depolarizing or hyperpolarizing conditioning current of $100 \mathrm{~ms}$ of $20 \%$ or $40 \%$ of the current for an unconditioned target response), I/V relation (relation between the magnitude of a $200 \mathrm{~ms}$ duration conditioning current, varying from $50 \%$ depolarizing to $100 \%$ hyperpolarizing, and the threshold at its end), recovery cycle (time-course of the threshold changes after a supramaximal conditioning stimulus eliciting action potentials). 
The following parameters were determined: threshold for an unconditioned target response of $50 \%$ (stimulus current required to evoke a CMAP of $50 \%$ of maximal), rheobase (slope of the Qt relation), strength-duration time constant (SDTC; absolute value of the x-intercept of the Qt relation), TEd90-100 (threshold decrease at the end of the $40 \%$ depolarizing conditioning stimulus), TEd40-60 (threshold decrease at 40 $60 \mathrm{~ms}$ of the $40 \%$ depolarizing conditioning stimulus), TEdpeak (maximal threshold decrease during $40 \%$ depolarizing conditioning stimulus), S2-accommodation (difference between TEdpeak and TEd90-100), accommodation half-time (time between the onset of the conditioning stimulus and the time point where threshold decrease is halfway between TEdpeak and TEd90-100), TEh90-100 (threshold increase at the end of the $40 \%$ hyperpolarizing conditioning stimulus), fanning (sum of the absolute values of TEd90-100 and TEh90-100), resting I/V slope (slope between $-10 \%$ and $+10 \%$ conditioning stimuli), minimal I/V slope (smallest slope in the hyperpolarizing part of the I/V curve), hyperpolarizing I/V-slope (slope between $100 \%$ and $80 \%$ hyperpolarizing conditioning stimuli), refractoriness at $2 \mathrm{~ms}$ (threshold change at the conditioning-test interval of $2 \mathrm{~ms}$ ), refractory period (time between conditioning stimulus and return of threshold to baseline), superexcitability (lowest threshold after refractory period) and subexcitability (highest threshold after superexcitability).

Primary endpoints were repeatability of these variables as assessed by Cronbach's alpha, and effects of riluzole and retigabine on the variables compared to placebo.

\section{Controls}

In a separate study, we also investigated excitability by the same methods in eighteen age-matched healthy controls ( 9 men, median age 53 years, range $35-71$ ) who had no neurological symptoms and did not use medication. 


\section{ALSFRS-R}

The ALSFRS-R(42) evaluating disability in patients with ALS was performed at baseline and approximately 3 months after the first dose. This revised version of the ALSFRS, which incorporates additional assessments of dyspnea, orthopnea, and the need for ventilatory support, retains the properties of the original scale and shows strong internal consistency and construct validity. The rating scale is a validated, reliable, rating instrument for monitoring the progression of disability in patients with ALS. $(43,44)$

\section{Data management}

All data were stored in a clinical trial database (Promasys, Omnicomm Inc., Fort Lauderdale, USA) and checked for accuracy and completeness. We performed a blinded data review before code-breaking and analysis according to a standard procedure at CHDR.

\section{Statistical analysis}

Pharmacokinetic parameters, including maximum plasma concentration $\left(\mathrm{C}_{\max }\right)$ and time to $\mathrm{C}_{\max }\left(\mathrm{T}_{\max }\right)$, were determined by standard non-compartmental methods using $R$ software version 3.4.0. Test-retest reliability of the primary endpoints was assessed by Cronbach's alpha, where a sample of 18 subjects was considered to be sufficient. An interim analysis evaluating between-day repeatability by Cronbach's alpha was performed after 12 patients had completed the study. A value of at least 0.7 (acceptable repeatability) was needed for at least one of the variables that were deemed most relevant -- refractoriness at 2 ms, SDTC, superexcitability, TEd40-60 or TEd90-100 -- to proceed with the final 6 patients. To establish whether significant 
treatment effects could be detected on the excitability parameters, a mixed model analysis of covariance was used with treatment, time and treatment by time as fixed factors and subject, subject by treatment and subject by time as random factors, and the average baseline measurement as covariate. The Kenward-Rogers approximation was used to estimate denominator degrees of freedom and model parameters were estimated using the restricted maximum likelihood method. To determine treatment effects on excitability parameters the estimated averages of both post-dose time points for each treatment were compared with placebo. Missing data were not imputed, but were estimated within the statistical model. Residual $Q-Q$ plots were used to check the assumption of normality of the error term in the mixed effects model together with the Shapiro-Wilk test for normality. Parameters violating the assumption of normality were log-transformed and after the analysis were backtransformed so that the results can be interpreted as a percentage change. The excitability variables of ALS patients at the first, pre-dose, visit were compared with those obtained in the healthy controls by unpaired Student's t-test. The correlation between the difference in ALSFRS-R scores (at baseline and at 3 months) and the first visit pre-dose excitability parameters was calculated using Spearman correlation. Significance level was set at $p<0.05$ and $95 \%$ confidence intervals of the estimated difference between the treatment and placebo groups are presented. All calculations were performed using SAS for windows V9.4 (SAS Institute, Inc., Cary, NC, USA). 


\section{Study Highlights}

- What is the current knowledge on the topic?

Patients with amyotrophic lateral sclerosis show increased membrane excitability in peripheral and central motor neurons, which may be a relevant factor leading to motor neuron damage. Current approved treatment with riluzole decreases hyperexcitability, indicating this could be a therapeutic target.

- What question did this study address?

The aim of this study was to validate peripheral motor nerve excitability-testing as biomarker of hyperexcitability and assess effects of riluzole and retigabine in patients with amyotrophic lateral sclerosis.

- What does this study add to our knowledge?

Between- and within-day repeatability was good for most excitability testing parameters and a single dose of retigabine, but not riluzole normalized several relevant markers indicating hyper-excitability in these patients.

- How might this change clinical pharmacology or translational science? Peripheral nerve excitability could be a very useful and quick, non-invasive biomarker to test for potential treatments for ALS, and measure treatment efficacy. Retigabine may be such a treatment reversing hyper-excitability, justifying long-term studies looking at impact on disease progression and survival. 


\section{Acknowledgements}

The authors would like to thank Biogen for their financial support, which funded the study.

\section{Author contributions}

J.A.A.C.H. and M.K. wrote the manuscript; J.A.A.C.H., M.K., B.T.H.M.S., L.vdB., T.A.F., H.F., and G.J.G. designed the research; J.A.A.C.H., M.K., and B.T.H.M.S.

performed the research; J.A.A.C.H., M.K., B.T.H.M.S., and D.Z. analyzed the data. 
(1) Bae, J.S., Simon, N.G., Menon, P., Vucic, S. \& Kiernan, M.C. The puzzling case of hyperexcitability in amyotrophic lateral sclerosis. J Clin Neurol 9, 65-74 (2013).

(2) Kanai, K. et al. Altered axonal excitability properties in amyotrophic lateral sclerosis: impaired potassium channel function related to disease stage. Brain 129, 953-62 (2006).

(3) Park, S.B., Kiernan, M.C. \& Vucic, S. Axonal Excitability in Amyotrophic Lateral Sclerosis : Axonal Excitability in ALS. Neurotherapeutics 14, 78-90 (2017).

(4) Vucic, S. \& Kiernan, M.C. Axonal excitability properties in amyotrophic lateral sclerosis. Clin Neurophysiol 117, 1458-66 (2006).

(5) Geevasinga, N., Menon, P., Howells, J., Nicholson, G.A., Kiernan, M.C. \& Vucic, S. Axonal ion channel dysfunction in c9orf72 familial amyotrophic lateral sclerosis. JAMA Neurol 72, 49-57 (2015).

(6) Menon, P., Kiernan, M.C. \& Vucic, S. ALS pathophysiology: insights from the splithand phenomenon. Clin Neurophysiol 125, 186-93 (2014).

(7) Kanai, K. et al. Motor axonal excitability properties are strong predictors for survival in amyotrophic lateral sclerosis. J Neurol Neurosurg Psychiatry 83, 734-8 (2012).

(8) Shibuya, K. et al. Increased motor axonal persistent sodium currents predict rapid functional declines in amyotrophic lateral sclerosis. Neurol Clin Neurosci 4, 108-11 (2016).

(9) Krarup, C. Lower motor neuron involvement examined by quantitative electromyography in amyotrophic lateral sclerosis. Clin Neurophysiol 122, 414-22 (2011).

(10) Kuwabara, S. \& Misawa, S. Axonal ionic pathophysiology in human peripheral neuropathy and motor neuron disease. Curr Neurovasc Res 1, 373-9 (2004).

(11) de Carvalho, M. Why is ALS so excited? Clin Neurophysiol 122, 1689-90 (2011).

(12) Wainger, B.J. et al. Intrinsic membrane hyperexcitability of amyotrophic lateral sclerosis patient-derived motor neurons. Cell Rep 7, 1-11 (2014).

(13) Vucic, S. et al. Riluzole exerts central and peripheral modulating effects in amyotrophic lateral sclerosis. Brain 136, 1361-70 (2013).

(14) Tomlinson, S.E., Tan, S.V., Kullmann, D.M., Burke, D., Hanna, M.G. \& Bostock, H. Axonal Excitability Changes in Genetic Neuronal Ion Channel Disorders. Journal of the Peripheral Nervous System 14, 144-5 (2009).

(15) Agency, E.M. SmPC Rilutek. $<$ http://www.ema.europa.eu/docs/en GB/document library/EPAR Product Information/human/000109/WC500056586.pdf> (2018).

(16) Gunthorpe, M.J., Large, C.H. \& Sankar, R. The mechanism of action of retigabine (ezogabine), a first-in-class $\mathrm{K}+$ channel opener for the treatment of epilepsy. Epilepsia 53, 412-24 (2012).

(17) Huang, C.J. et al. Characterization of voltage-gated sodium-channel blockers by electrical stimulation and fluorescence detection of membrane potential. Nat Biotechnol 24, 439-46 (2006).

(18) Bostock, H. \& Baker, M. Evidence for two types of potassium channel in human motor axons in vivo. Brain Res 462, 354-8 (1988).

(19) Reid, G., Scholz, A., Bostock, H. \& Vogel, W. Human axons contain at least five types of voltage-dependent potassium channel. J Physiol 518 ( Pt 3), 681-96 (1999). 
(20) Schwarz, J.R. et al. KCNQ channels mediate IKs, a slow K+ current regulating excitability in the rat node of Ranvier. J Physiol 573, 17-34 (2006).

(21) Devaux, J.J., Kleopa, K.A., Cooper, E.C. \& Scherer, S.S. KCNQ2 is a nodal K+ channel. J Neurosci 24, 1236-44 (2004).

(22) Tatulian, L., Delmas, P., Abogadie, F.C. \& Brown, D.A. Activation of expressed KCNQ potassium currents and native neuronal $\mathrm{M}$-type potassium currents by the anticonvulsant drug retigabine. J Neurosci 21, 5535-45 (2001).

(23) Wua, Y.J. \& Dworetzky, S.I. Recent developments on KCNQ potassium channel openers. Curr Med Chem 12, 453-60 (2005).

(24) Kiernan, M.C. \& Bostock, H. Effects of membrane polarization and ischaemia on the excitability properties of human motor axons. Brain 123 Pt 12, 2542-51 (2000).

(25) Bostock, H. \& Rothwell, J.C. Latent addition in motor and sensory fibres of human peripheral nerve. J Physiol 498 ( Pt 1), 277-94 (1997).

(26) Lang, P.M., Fleckenstein, J., Passmore, G.M., Brown, D.A. \& Grafe, P. Retigabine reduces the excitability of unmyelinated peripheral human axons.

Neuropharmacology 54, 1271-8 (2008).

(27) Kiernan, M.C., Guglielmi, J.M., Kaji, R., Murray, N.M. \& Bostock, H. Evidence for axonal membrane hyperpolarization in multifocal motor neuropathy with conduction block. Brain 125, 664-75 (2002).

(28) Kiernan, M.C., Mogyoros, I. \& Burke, D. Differences in the recovery of excitability in sensory and motor axons of human median nerve. Brain 119 ( Pt 4), 1099-105 (1996).

(29) Corbin-Leftwich, A., Mossadeq, S.M., Ha, J., Ruchala, I., Le, A.H. \& Villalba-Galea, C.A. Retigabine holds KV7 channels open and stabilizes the resting potential. J Gen Physiol 147, 229-41 (2016).

(30) Fleckenstein, J., Sittl, R., Averbeck, B., Lang, P.M., Irnich, D. \& Carr, R.W. Activation of axonal Kv7 channels in human peripheral nerve by flupirtine but not placebo therapeutic potential for peripheral neuropathies: results of a randomised controlled trial. J Transl Med 11, 34 (2013).

(31) Sittl, R., Carr, R.W., Schwarz, J.R. \& Grafe, P. The Kv7 potassium channel activator flupirtine affects clinical excitability parameters of myelinated axons in isolated rat sural nerve. J Peripher Nerv Syst 15, 63-72 (2010).

(32) Baker, M., Bostock, H., Grafe, P. \& Martius, P. Function and distribution of three types of rectifying channel in rat spinal root myelinated axons. J Physiol 383, 45-67 (1987).

(33) Bellingham, M.C. A review of the neural mechanisms of action and clinical efficiency of riluzole in treating amyotrophic lateral sclerosis: what have we learned in the last decade? CNS Neurosci Ther 17, 4-31 (2011).

(34) Zoccolella, S. et al. Riluzole and amyotrophic lateral sclerosis survival: a populationbased study in southern Italy. Eur J Neurol 14, 262-8 (2007).

(35) Schuster, J.E., Fu, R., Siddique, T. \& Heckman, C.J. Effect of prolonged riluzole exposure on cultured motoneurons in a mouse model of ALS. J Neurophysiol 107, 484-92 (2012).

(36) Jablonski, M.R. et al. Selective increase of two $A B C$ drug efflux transporters at the blood-spinal cord barrier suggests induced pharmacoresistance in ALS. Neurobiol Dis 47, 194-200 (2012). 
(37) Brooks, B.R., Miller, R.G., Swash, M., Munsat, T.L. \& World Federation of Neurology Research Group on Motor Neuron, D. El Escorial revisited: revised criteria for the diagnosis of amyotrophic lateral sclerosis. Amyotroph Lateral Scler Other Motor Neuron Disord 1, 293-9 (2000).

(38) Le, L.A. et al. Single- and multiple-dose pharmacokinetics of riluzole in white subjects. J Clin Pharmacol 37, 820-7 (1997).

(39) Drenthen, J., Blok, J.H., van Heel, E.B. \& Visser, G.H. Limb temperature and nerve conduction velocity during warming with hot water blankets. J Clin Neurophysiol 25, 104-10 (2008).

(40) Franssen, H. \& Wieneke, G.H. Nerve conduction and temperature: necessary warming time. Muscle Nerve 17, 336-44 (1994).

(41) Geerlings, A.H. \& Mechelse, K. Temperature and nerve conduction velocity, some practical problems. Electromyogr Clin Neurophysiol 25, 253-9 (1985).

(42) Cedarbaum, J.M. et al. The ALSFRS-R: a revised ALS functional rating scale that incorporates assessments of respiratory function. BDNF ALS Study Group (Phase III). J Neurol Sci 169, 13-21 (1999).

(43) Kaufmann, P. et al. Excellent inter-rater, intra-rater, and telephone-administered reliability of the ALSFRS-R in a multicenter clinical trial. Amyotroph Lateral Scler $\mathbf{8}$, 42-6 (2007).

(44) Kollewe, K., Mauss, U., Krampfl, K., Petri, S., Dengler, R. \& Mohammadi, B. ALSFRS-R score and its ratio: a useful predictor for ALS-progression. J Neurol Sci 275, 69-73 (2008). 


\section{Figure legends}

Figure 1 Study flow diagram

Figure 2 Pharmacokinetics of riluzole and retigabine. Mean with standard deviation concentration-time profiles in plasma per treatment (TRT).

Figure 3 Mean excitability recordings pre-dose (black), and 1.5 hours (red) and 6 hours (green) after a single dose of $100 \mathrm{mg}$ riluzole (i) and $300 \mathrm{mg}$ retigabine (ii). (A) current/voltage relationship, (B) strength-duration properties plotted as stimulus charge versus stimulus duration, (C) threshold electrotonus, (D) recovery cycle. Asterixes indicate significant treatment effects on thresholds (see Table 3).

Figure 4 Change from baseline plot of the treatment effect on $(A)$ refractoriness at 2 ms, (B) strength duration time constant and (C) hyperpolarizing I/V-slope. $\mathrm{Cl}$, Confidence interval; Retigabine300, retigabine $300 \mathrm{mg}$ treatment; Riluzole100, riluzole $100 \mathrm{mg}$ treatment. 